\title{
IL PIRANDELLISMO DI BUZZATI (Seconda Parte)
}

\section{Franco Zangrilli}

\begin{abstract}
Pirandello's influence on contemporary writers is impossible to measure. Pirandellismo is evident in Buzzati's short stories, novels and plays. Buzzati operates with a pirandellian humour which underlines the tragic aspects of life. Many characters in Buzzati's works, including the image of the mother depicted in II deserto dei Tartari, show a vivd pirandellian personality, and they are entroped in a continuous process of the distintegration of the "I". Buzzati treats many pirandellian themes, for example the impossibility to communicate with the others, the contrast between life and art, the relativism of reality, with fantastic tools. The originality of Buzzati's pirandellismo can be seen in "L'errneo fu" which, like II fu Mattia Pascal, presents an individual who sees the flux of life from the world of death, or in "Sette messaggeri" where, as in Enrico IV, a complex discussion takes place regarding the function of language in time and in space.
\end{abstract}

Molti racconti pirandelliani presentano personaggi, umoristici e non, che fanno uso estroso del fantastico, dipingendo atmosfere misteriose, casi enigmatici, fatti inverosimili, situazioni ora favolose ora magiche ora oniriche (ad es. "Soffio", "Il Vecchio Dio", "La casa dell'agonia"). Specie nell'ultima fase creativa di Pirandello essi diventano veri e propri racconti surrealisti (ad es. Berecche e la guerra, Una giornata). Buzzati riflette attentamente su quest'ultimo Pirandello e i suoi 
racconti mostrano varie sedimentazioni pirandelliane. Spesso ambedue gli scrittori partono da un dato realistico per arrivare al racconto fantastico-surrealistico, cercando la possibilità di rendere credibile l'assurdo, di scrutare la realtà alla rovescia, di vedere la dimensione alogica di un'immagine e di esplorare paradossalmente l'opposizione tra realtà e illusione, tra essere e apparire, tra noto ed ignoto, ecc., come ad esempio si avverte nel racconto "La grande biscia" in cui si raffigura un ambiguo avvistamento di un serpente ora presente ora assente, ora vicino ora lontano, ora visibile ora invisibile, che emblemizza il mistero della vita, oltre a quell'idea pirandellana che la vita si vive senza vederla (CB, 87-93). I loro protagonisti si trovano imprigionati frequentemente in meccanismi caotici, in vicende esotiche, da cui non possono liberarsi, diventando vittime del caso d'anormalità, e finanche di se stessi. Il protagonista del racconto surrealista o poliziesco o metaletterario o di altro genere di Pirandello e di Buzzati è sempre un'allegoria di una coscienza che simultaneamente vive valori umani e metafisici, inquieta, travagliata, angosciata anche perchè è costantemente alla ricercare di qualcosa inafferrabile e della propria identità. In parecchi aspetti il racconto surrealista di Pirandello prepara e influisce su quello di Buzzati, come si potrebbe constatare con un raffronto tra "Soffio" di Pirandello e "Il borghese stregato" di Buzzati che dipingo una personalità dimezzata alquanto complessa del personaggio.

Infatti il pirandellismo di Buzzati si fa vibrante quando nei suoi racconti si viene a trattare il tema del doppio con incessante ricorrenza e freschezza ripetitiva, con intento di renderlo un referente del mistero o di aprirlo a un composito discorso cronotopico (ad es. "Quando l'ombra scende", "Cacciatori di vecchi", "Un verme in casa"), con uno stile umoristico che sa farsi molto colorito (ad es. "Viaggio agli inferi del secolo"), come mostra il racconto dialogato "La soffitta" in cui le due anime interiori del protagonista, la buona e la cattiva, si scontrano, in modo molto simile a quelle del protagonista pirandelliano (ad es. "Non è una cosa seria", "Quand'ero matto", "Stefano Giogli, uno e 
due") ${ }^{1}$, e con una folla di personaggi che presentano sempre nuovi lineamenti, in un continuo scambio, giuoco, e creazione di ruoli, specie di scissione e di ricomposizione dell'identità. Onde, come in Pirandello, si sfrutta il topos del travestimento (ad es. "Alfredo Brilli commercialista" di Buzzati e "La patente" di Pirandello ${ }^{2}$ ), dell'impostura (ad es. "Lo scandolo a via Sesostri" di Buzzati e "La berretta di Padova" di Pirandello), del costruirsi e quindi del fingere di essere un altro (ad es. "L'assalto al grande convoglio" di Buzzati e "Un matrimonio ideale" di Pirandello"), dell' incontro di due amici anime gemelle ma che non si riconoscono ("Un verme in casa" di Buzzati e "Amicissimi" di Pirandello).

E se lo spiritismo nei racconti siciliani e non di Pirandello si adopera per raffigurare vicende di crisi esistenziali e della personalità dell'individuo (ad es. "La casa del Granella", "Personaggi", "Lo spirito maligno", "Dal naso al cielo", Il fu Mattia Pascal), anche nei racconti buzzatiani si utilizza in questa accezione, in misura pirandelliana per configurare una miriade di elementi della problematica dell'io, sempre al centro di una perenne metamorfosi, di processi di mutamenti che ricostruiscono la sembianza del suo doppio (ad es. Esperimenti di magia).

\footnotetext{
Va notato che il personaggio pirandelliano, da Perazzetti a Baldovino, disquisisce filosoficamente delle due anime in noi, tanto che l'una si identifica con Dio e l'altra con il Diavolo. Per ulteriori informazioni su questo argomento si veda il mio studio Il bestiario di Pirandello, Pesaro, Metauro Edizioni, 2001: 127-147.

2 Nel suo saggio L'umorismo L. Pirandello, Saggi, poesie, scritti vari, Milano, Mondadori, 1973: 153, dice: "ciascuno si racconcia la maschera come può - la maschera esteriore -. Perchè dentro poi c'è l'altra, che spesso non s'accorda con quella di fuori”'.
} 
In "Un pittore morto da 70 anni ha dipinto un paesaggio a Torino" figura un personaggio stregone, Rol, con straordinarie doti intellettuali e con una personalità profondamente ambigua ${ }^{3}$. Crede che lo spirito dei "morti" rimanga tra noi manifestando il suo doppio, che si reincarni in individui per operare attraverso loro, e che così ripete "cose che aveva fatto durante la vita" (MI, 52-53). Queste sue credenze e tratti della sua composita identità vengono elaborati, tanto che l'autore ritorna a gettar luce sulla sua "faccia" cangiante di mago: "Qualcuno l'ha definita da bon vivant. Non è vero. Potrebbe essere quella di un gurù indiano. Ma potrebbe anche appartenere a un chirurgo, a un vescovo, a un tenero bambino" o a un artefice di "esperimenti" artistici (MI, 51-52).

D. Buzzati, I misteri d'Italia, Milano, Mondadori, 1978: 51. D'ora in poi le iniziali MI e il numero della pagina nel testo rimanderanno a questa edizione. 
Nel suo appartamento Rol si diletta facendo "esperimenti di pittura". E li realizza con la partecipazione del pittore francese François-Auguste Ravier, deceduto nel 1895 a Lione. Lo "spirito" di Ravier, manifestandosi ed esprimendosi artisticamente attraverso Rol, combina un complicato giuoco di specchi e di metamorfosi, di riflessioni e di rimandi, di doppiezze e di dimidiamenti (MI, 57-58). Pirandellianamente, Ravier e Rol, svolgono ruoli reciproci, interscambiabili, identici. L'uno è il corpo e l'altro l'ombra; l'uno è la maschera e l'altro il volto; l'uno è la parte conscia e l'altro la parte inconscia della coscienza. Sono due in uno. Il processo di (ri)unificazione-scissione fa emergere una quantità di motivi dualistici, soprattutto il motivo dell'individuo morto-vivo, che come si è visto è preponderante nei racconti dei nostri scrittori, e il motivo del tempo passato-presente, ma che gradatamente si trasforma in un eterno presente che appartiene solo all'arte (della pittura), idea sottolineata da varie opere pirandelliane ("L'uccello impagliato", "Effetti di un sogno interrotto", "Colloqui coi personaggi"). Come vari personaggi pittori di Pirandello (ad es. "Effetti di un sogno interrotto" "Il coppo", L'innesto), il pittore Rol-Ravier diventa tanto la parte dimezzata quanto il doppio del Buzzati pittore. Attraverso la doppiezza del pittore ottocentesco-novecentesco, Buzzati confessa elementi della sua passione di pittore, di uomo che, come Pirandello, ha sempre amato di disegnare e di dipingere quadri ${ }^{4}$. E proprio mentre Buzzati descrive l'abilità di Rol-Ravier di dipingere un quadro di "grazioso paesaggio", le fasi dello svolgimento del rito pittorico del personaggio, l'atto della creazione che conduce a stati beatificanti, affabula e discute parecchie idee che vive nei riguardi dell' arte della pittura, dalla scelta dei soggetti a quella dei colori, a quella della "tecnica", e che rivelano certi sui gusti d'estetica (MI, 56, 58-59).

4 Sul rapporto di Pirandello con la pittura si veda A. Alessio, Pirandello pittore, Agrigento, Edizioni del Centro Nazionale di Studi Pirandelliani, 1984, e E. Cecchi, Pirandello pittore, in Omaggio a Pirandello, a cura di L. Sciascia, Milano, Bompiani, 1986. 
Soprattutto verso lo scioglimento della narrazione, all'immagine seria del pittore Rol(-Ravier-Buzzati) si oppone quella poco seria del mago stregone quando si trova in uno stato di trance, burlona, scherzosa, ilare, che si intrattiene e intrattiene prendendo in giro gli astanti e al tempo stesso svelando loro profonde verità e filosofie, combinando incantevoli apparizioni, sedute spiritiche, giuochi e trucchi magici, prodigiosi, misteriosi, ammalianti (MI, 58-59). Un mago che in molti aspetti rassomiglia al "poeta mago" Cotrone dei Giganti della Montagna che domina gli spiriti del mondo magico degli Scalognati e che magicamente si sdoppia soprattutto quando disquisisce del suo passato nell' "aiuola" che ci rende tanto "feroci".

Non diversamente dal Pirandello che predilige filtrare le esperienze personali nella rappresentazione grottescamente fantastica (ad es. "Dialogo tra il Gran Me e il piccolo me"), Buzzati si mette faccia a faccia con se stesso, e pur con atteggiamenti narcisistici, cercando di scrutare e di analizzare tutte le possibili sfaccettature del suo io e quindi del suo personaggio in crisi.

Nel racconto "L'ubiquo", che si muove con andamenti diaristici di viva confessione, il personaggio narratore(-Buzzati) riflette sulla sua professione di giornalista. L'anormale qui si identifica con il processo dell'ubiquità che egli sperimenta senza poterlo controllare. Le oscillazioni interiori di "parlarne o no al [suo] direttore" ingigantiscono la crisi e si immagina, con atteggiamento inquisitorio, una vittima del direttore che fa del tutto per salvaguardare le apparenze sociali dell'istituto da lui guidato, il prestigio e la "bella figura" del giornale (IC, 233, 241). Il fenomeno dell'ubiquità è un fenomeno di dimidiamento che ripetendosi travolge il personaggio, e conduce il racconto a colorirsi sempre più di "realismo magico". Essendo in casa, a un tratto si ritrova magicamente ed inconsciamente in redazione del suo giornale, il "Corriere" (IC, 236). E apprende che di colpo riesce a spostarsi da un luogo all'altro "con una velocità superiore a quella della luce" (IC, 237). Tuttavia Buzzati sfrutta le immagini della celerità della luce e del processo dell'ubiquità per sottolineare la perenne 
scissione dell'io, dell'individuo travolto dal vortice di una professione che richiede di essere presente in più luoghi e di svolgere più mansioni. Umoristicamente il personaggio scopre che come una sorta di dio onnipresente può essere ovunque al tempo stesso annullando le dimensioni del tempo-spazio, e che come una sorta di scaltro detective può pescare ogni notizia nel globo (IC, 238-239). Nella rappresentazione si indugia a lungo su quest'azione inverosimile del giornalista. E di conseguenza il grottesco si accende in modo sfolgorante, al punto che viene a parodiare la velocità irrazionale con cui oggi camminano le notizie e quindi la difficoltà da parte del destinatario di assimilarle, ad ironizzare sulla figura stravagante del giornalista che sfrutta la potenza magica dell'ubiquità (IC, 239-240). Per Pirandello e per Buzzati il "potere" giornalistico è enorme, e si usa non sempre per cambiare in meglio la vita sociale ${ }^{5}$. Come $\mathrm{i}$ giornalisti configurati in Suo marito di Pirandello, quello dell'"Ubiquo" protegge con grande risolutezza e segreto il suo "potere" (IC, 240-241). La preoccupazione di perderlo acuisce il panico e la paranoia, presenta un Buzzati scivolato in una estrema crisi d'identità, nell'ambito della schizofrenia che sembra aggravarsi con la meditazione del suicidio: "Addio Dino Buzzati. Una rivoltella nella nuca, o una bella dose di cianuro non te la toglierebbe più nessuno" (IC, 241).

In altri racconti il pirandellismo buzzatiano si approfondisce perchè il personaggio in crisi si giuoca su una scacchiera di specchi, intrecciando freschi motivi del raddoppiamento e dello sdoppiamento della personalità ( ad es. "Il sosia", "Il fratello cambiato", "Quando l'ombra scende", "Grandezza dell'uomo”).

5 Per ulteriori informazione su questo argomento si veda il mio studio Pirandello $e$ il giornalismo, Roma-Caltannissetta, Salvatore Sciascia Editore, 2003. 
Nel racconto "L'alienazione" Buzzati scrive una lunga lettera al direttore del "Corriere", come sempre riportata in corsivo all' incipit, lamentandosi di un intenzionale plagio: "sul giornale da lei diretto, $e$ nel quale lavoro da immemorabile tempo, è uscito un articolo firmato col mio nome. Ma non l'ho scritto io" ${ }^{6}$. Se da una parte Buzzati è molto critico dell'operare dei giornalisti, dall'altra parte elogia l'articolo redatto dal collega che usa la sua firma ("era un buon articolo, lo devo ammettere onestamente. E vi ho riscontato uno stile abbastanza simile al mio" ND, 255). Da qui scatta l'escamotage dell'anonimia e dell'omonimia per tessere una distinta vicenda pirandelliana. L' anonimo collega è un enigmatico intruso, la "maschera dimenticata", il doppio di Buzzati. Il complicato giuoco di disintegrazione della personalità si dilata su dimensioni cronotopiche antitetiche (come succede in altre fabule buzzatiane quale "Quando l'ombra scende"). In modo poliziesco l'uno perseguita e fugge dall'altro, persino nei misteriosi e labirintici corridori del palazzo del "Corriere della Sera" (ND, 255-256). L'intruso rappresenta a tanti livelli il conscio e l'inconscio di Buzzati, e una sorta di specchio in cui i colleghi lo vedono esattamente com'è, con i suoi umori, pensieri, lineamenti, che causa epifanie, sconvolgimenti, ed estraniazioni a diverse persone e allo stesso Buzzati ("Questo Buzzati che ha firmato non so chi sia" ND, 256). L'intruso simboleggia una larva diabolica che si incarna nella personalità di Buzzati con lo scopo di rendergli infelice la vita di giornalista, oltre a un'ombra del Buzzati che ama (auto)dipingersi e (auto)elogiarsi, ombra ora di vecchio ora di giovane ora di demiurgo che medita sull' "onda del tempo che a poco a poco ci trasmuta e ci divora":

Mi domando se questo Dino Buzzati, che non conosco, non sia alle volte lo strumento, forse

D. Buzzati, Le notti difficili, Milano, Mondadori, 1998: 254. D'ora in poi le iniziali ND e il numero della pagina nel testo rimanderanno a questa edizione. 
inconsapevole, di un processo fatale. Non sia l'incarnazione nuova di me stesso, destinato a prendere il mio posto [...] L'avete chiamato a collaborare questo nuovo Dino Buzzati della malora, probabilmente giovanissimo, potrebbe essere perfino un segno di riguardo. Quasi a garantirmi che la mia bandierina, sia pure tenuta da altre mani, continuerà a sventolare.

Certo, mi piacerebbe conoscerlo. Non penso sia un demoniaco William Wilson, uguale a me anche fisicamente, venuto al mondo per dannarmi [...] Questo Dino Buzzati esiste [...] Pare che sia un ragazzotto di ventisette, ventott'anni. Molto per bene. Coltissimo. (ND, 256)

Come nella produzione di Pirandello (ad es. "Lo spirito maligno", "L'ombra del rimorso", Il fu Mattia Pascal), in quella buzzatiana l'ombra (ad es. "L'ubiquo", "L'erroneo fu”, “Quando 1'ombra scende"), diventa un clichè che denota l'identità frantumata, sdoppiata, scissa; il buio dell'inconscio; l'incubo o l'ossessione che ci tortura; il nostro passato lontano, ma che spesso si identifica con il periodo della fanciullezza, che si recupera con ricordi di triste malinconia, come testimonia la vicenda di un uomo maturo che si rivede bambino, in "Ritorno" di Pirandello e in "Quando scendo l'ombra" di Buzzati. L'ombra che ci pedina ed è sempre attaccata al nostro corpo allegorizza la morte che non si stanca di perseguitare la vita, di cui sono rivelatrici "La morte addosso" di Pirandello e "Ombra del Sud" di Buzzati, vuol essere il sosia dell'eterna annullatrice e quindi di un tempo che ci trascina verso il nulla (ad es. "La maschera dimenticata" di Pirandello e "Storia personale" di Buzzati). L'aspetto larvale dell'ombra rispecchia il mistero che circonda il mondo della morte o la misteriosa personificazione della morte che va e viene toccando 
questo o quello, quasi come messaggera del fato (cfr. ad es. L'uomo dal fiore in bocca di Pirandello e Piccola passeggiata di Buzzati); a volte l'ombra della morte contrasta umoristicamente con quella di Dio (ad es. "Il vecchio Dio" di Pirandello e "Un dio scende in terra" di Buzzati). Anche il Buzzati dell' "Alienazione" è convinto di essere un ombra evanescente nel gran mare della vita. Quando è a lavoro ha la sensazione che i colleghi non lo salutino perchè non lo riconoscono, di essere un estraneo che si sta dissolvendo in "qualcosa di inconsistente" (ND, 257, facendo ricordare il dissoversi nelle cose animate e disanimate del personaggio pirandelliano - ad es. "Di sera, un geranio", "Canta l'Epistola", "La mano del malato povero" -). E tragicomica è la scena in cui il dottor Dino Buzzati scopre che in redazione il suo posto è stato usurpato da un altro Dino Buzzati, perfettamente simile a lui ma "totalmente" forestiero e diavolesco:

Stamane sono andato al giornale [...] I fattorini all'ingresso non mi hanno salutato. Anzi mi hanno chiesto: "Scusi, signore desidera?"

"Come, desidero? Sono Buzzati, no? Che scherzi sono questi?"

"Il dottor Buzzati" ha risposto il capo portiere "è nel suo ufficio. Se lei desidera parlargli, firmi qui, la prego, il modulo" [...]

Là, nello studio, dietro allo scrittoio, sono seduto io. Ma non giovane. Anzi. Della mia stessa età. A me totalmente straniero. Odioso. Mi sorride: "In che cosa posso...?" (ND, 257)

Ma, come nello "Spirito maligno", in "Ritorno", e in altri racconti pirandelliani, in "Riservatissima per il signor direttore" il tema della doppiezza e del dimidiamento è più strettamente connesso a quello del diavolo. Di nuovo si ha un Buzzati che scrive una lettera al direttore del "Corriere", ma al quale vuole confessare una strana storia iniziata all'epoca del suo apprendistato giornalistico. Il flashback, costellato da 
pennellate di (auto-)ironia, conduce a una dettagliata rievocazione di quando "era semplice cronista" e il desiderio di affermarsi nella carriera era fortissimo; nel periodo di praticantato l'ardente passione per il giornalismo è identica alla struggente ambizione di scrivere racconti (IC, 87-90).

Inaspettatamente in redazione si vede apparire lo zio di un suo vecchio amico. Avendo la debolezza di scrivere racconti e romanzi Ileano Bissàt gli chiede di aiutarlo a pubblicare sul "Corriere". Se Bissàt vede il Buzzati giornalista un uomo di potere, Buzzati tra i tanti giornalisti dell'azienda si vede un "nessuno"; il dialogo rovesciando i ruoli assume le movenze dell'intervista in cui Bissàt appare un corrispondente che pone importanti domande e osservazioni che vanno nel cuore delle cose e delle ambizioni dell'intervistato, e che incoraggia il Buzzati deluso di aver intrapreso tale professione:

"Lei lavora in un grande giornale, ha relazioni, ha appoggi, ha autorità, lei potrebbe..."

"Io? Ma io sono l'ultima ruota del carro. E poi il giornale non pubblica scritti letterari se non sono di grandi firme".

"Ma lei..."

"Io non firmo. Io sono un semplice cronista. Ci mancherebbe altro" [...]

"Ma le piacerebbe firmare?"

"Si capisce. A esserne capaci!"

"Eh, signor Buzzati, non si butti via così! Lei è giovane, lei ne ha del tempo dinanzi. Vedrà, vedrà. (IC, 89)

Il giovane cronista Buzzati si porta a casa il malloppo di manoscritti lasciati da Bissàt, ed accidentalmente una sera ne legge alcuni rimanendo folgorato perchè "assomigliano" alle sue cose: "Boia d'un mondo che roba. Era strana, era nuova, era bellissima [...] Corrispondeva maledettamente a me, mi assomigliava, mi dava il senso 
di essere io. Erano una per una le cose che io avrei voluto scrivere e invece non ero capace. Il mio mondo, i miei gusti, i miei odii. Mi piaceva da morire" (IC, 90). Così si scoprire che Buzzati diventa un critico fantastico della propria scrittura e che Bissàt è quello che il giovane Buzzati avrebbe voluto essere e non è stato, è l'altra parte della coscienza dimezzata di Buzzati, è il doppio di un Buzzati che ha superato e non sente più il fardello di quella "maledetta ambizione letteraria" (IC, 90). Nella narrazione i giuochi ossimorici, paradossali, ed umoristici di stampo pirandelliano si accumulano a dovizia e su moduli del fantastico, vengono portati all'estremo per imprimere l'assurdo dell'intreccio, e dipingono l'ambizioso Buzzati che viene fuori dalla fase del tirocinio stringendo il patto col diavolo. Cioè con un Bissàt satanico che sa indossare la maschera di un signore dalla personalità melliflua, "modesto, bonario, gentile", e la cui "umiltà estrema era la cosa che faceva più colpo" (IC, 88, 95). Come in "Ritorno", "Lo spirito maligno", "Il fumo", e altri racconti pirandelliani in cui si fa dominante la figura del diavolo, qui un vagliato sistema di immagini ricreano felicemente il mito mefistofelico che enfatizza la disintegrazione e la moltiplicazione della personalità. Bissàt simboleggia il demonio in noi, tutto ciò che di cattivo e di malizioso alberga nel nostro animo, incluso il sentimento spietato di realizzarci in una professione commettendo amoralità (come fanno certi giornalisti del romanzo pirandelliano Suo marito). È una sembianza larvale del diavolo che opera nella vita di ogni giorno. Sin dalla prima visita Bissàt rappresenta un drammatico scontro/incontro tra il passato e il presente della vita di Buzzati, fitto di serie osservazioni che prendono a bersaglio i critici letterari, fugate dalla nota del comico anche mentre si discute dell' "ignobile" contratto, ossia della volontà di Bissàt di cedere i suoi scritti al giovane Buzzati per una ragionevole remunerazione e che questi pubblicherà col proprio nome: 
"Senta, signore. Mi creda. Io ho soltanto bisogno di quattrini. Non ho ambizioni. Se scrivo è per puro passatempo [...] Io ho scritto, la firma la mette lei. Lei è giovane [...], io sono vecchio. Lanciare un vecchio non dà soddisfazione. Mentre i critici puntano volentieri sui ragazzi che debuttano" [...]

"Ma sarebbe una truffa, uno sfruttamento ignobile".

"Perchè? Lei mi paga. Io mi servo di lei come di un mezzo per piazzare la merce. Il conto torna [...] La rifornirò man mano. Mi guardi in faccia. Le pare che io sia un tipo capace di tradirla" [...]

"E se poi il giornale mi manda a fare un viaggio?"

"La seguirò". (IC, 92-93)

Stabilito il complotto Bissàt segue il giovane cronista Buzzati come un sosia. Il cronista a volte teme di ritrovarsi in balia di un sosia che potrebbe trascinarlo nell'ambito dello "scandolo" (IC, 93). Più il direttore del giornale e i colleghi giornalisti riconoscono l'alto valore dei suoi elzeviri, racconti e romanzi, più Bissàt diventa un sosia diabolicamente antagonista e nemico. Come si nota in certi racconti di Pirandello (ad es. "La maschera dimenticata", "La morta e la viva"), il motivo del sosia qui si sviluppa su vari piani, al punto che Bissàt appare ora la proiezione futuristica di Buzzati, ora "l'ombra del rimorso" del Buzzati che si è comportato scorretto con se stesso e con gli altri, ora la forza dei demoni interiori che domina la nostra vita (IC, 96-97, e cfr. "La moglie con le ali", "Piccola circe", Un amore ed altre opere di Buzzati). Infatti attraverso tante favole Pirandello e Buzzati suggeriscono che sono propri questi demoni interiori a trascinarci in vertiginosi processi di frantumazione, a condurci nell'ambito della perdita d'identità, a farci sentire uno, nessuno e centomila ${ }^{7}$.

7 Un sentimento sperimentato anche dal Buzzati adolescente come evidenziano alcune sue Lettera a Bambrilla, cit: 2002-203, specie quando ci confessa che "bisogna" cambiare personalità con gli altri, tanto che si deve apparire uno per un amico e un altro per un altro amico: "bisogna che finga d'essere diverso da quel che sono". 
Altri elementi di spersonalizzazione traspaiono dall' azione ripetitiva e rituale del personaggio, pirandelliano e buzzatiano, di mettersi davanti allo specchio. Lo specchio approfondisce le sue crisi ed i sui squilibri psicologici, lo presenta con un'identità instabile, bifronte, senza "qualità", e con volti sempre nuovi: anche il protagonista della "Notte" ci informa che "gli specchi [...] rimandano una faccia strana" (PS, 217). Lo specchio porta a sperimentare l'illusione e la delusione, la gioia e l'amarezza, l'amore e l'odio, la polarità di tanti sentimenti, come scoperte, rivelazioni, epifanie, oltre che inquietudini, angosce, tormenti; diviene il suo interlocutore facendo emergere intime confessione e menzogne, aspri dibattiti e diatribe, profonde oscillazioni, ambiguità, conflittualità, e tante filosofie (ad es. Il fu Mattia Pascal, Così è se vi pare, Trovarsi di Pirandello; e Teatro, Il colombre, Il crollo della Baliverna di Buzzati).

Al cospetto dello specchio combina smorfie e sorrisi umoristicamente acerbi, ma a volte connotanti l'intima felicità, come mostra Lucina della "Moglie con le ali" che guardandosi allo specchio sorride "come una stupidella" perchè è contenta della novità di avere un amante, ND, 318-319). Di fronte allo specchio apprende con stupore qualcosa di nuovo del proprio corpo, come l'improvviso mutamento degli aspetti somatici del volto o del naso: "Il mio naso era diritto, floscio e insignificante, adesso è piuttosto aquilino e nello stesso tempo rincagnato [...], adesso mi piaccio enormemente quando mi esamino nello specchietto" (IC, 456, e cfr. l'apertura di Uno, nessuno e centomila dell'agrigentino), sentendo in sè quegli "istinti del Dottor Jekyll e Mr. Hyde" (IC, 457) o in viva disarmonia ${ }^{8}$. Lo specchio metaforizza il senso tragico delle cose, anche quando testimonia la sua consapevolezza del tempo che passa, come avvertono tante opere dei nostri autori, dalle "poesie" a La signora Morli, una e

\footnotetext{
8 Si veda ad esempio D. Buzzati, "Autorimessa Erebus", in Il crollo della Baliverna, Milano, Mondadori, 1997: 74-80. D'ora in poi le iniziali CB e il numero della pagina nel testo rimanderanno a questa edizione.
} 
due, a Trovarsi di Pirandello, Il deserto dei Tartari dall'apertura che ritrae la partenza del protagonista per la Fortezza Bastiani ("vestì per la prima volta la divisa di tenente. Come ebbe finito, al lume di una lampada a petrolio si guardò nello specchio, ma senza trovare la letizia che aveva sperato [...] Ma in fondo - si accorse Giovanni Drogo - il tempo migliore, la prima giovinezza, era probabilmente finita. Così Drogo fissava lo specchio, vedeva uno stentato sorriso sul proprio volto, che aveva cercato di amare" DT, 23-24) alla chiusura che lo ritrae moribondo durante il viaggio di ritorno a casa ("Drogo scese dal letto e fu colto da un'onda di vertigine, che però lentamente si dissolse. Adesso era davanti allo specchio e guardava spaventato il proprio volto, giallo e consunto" DT, 236), Un verme in ministero: "MORALES Io ho passato ormai i cinquant'anni... FLORA Nooo! MORALES Eh, si vede, si vede. Al Professor Morales lo specchio non dice bugie. Porto gli occhiali, non sono certo bello"(T, 313), e altre opere buzzatiane più contraddistinte dal carattere umoristico del personaggio che si smarrisce ad esaminare la propria foto allo specchio, descritta con sintesi di sineddoche e d'immagini: "nello specchio c'è la mia figura, la mia faccia patita, i miei capelli grigi, ci sono anni consumati, tanti, la lunga strada (io camminavo a petto infuori, fiducioso di me, sicuro che ce l'avrei fatta da solo fino in fondo" (CB, 79-80). In Pirandello e in Buzzati lo specchio si fa una metafora di esplorazione, di scavo, di analisi, di un continuo ripiegamento della coscienza che vuole capire il senso della propria esistenza. Per cui spiccano i dettagli del tempo deformatore della nostra personalità (cfr. ad es. "Gli specchi”, SS, 71-72, e Trovarsi), e quelli del contrasto tra la giovinezza e la vecchiaia (cfr. ad es. CB, 78-79, e "Una giornata" di Pirandello); il personaggio femminile del dramma buzzatiano L'uomo che andrà in America commenta: "La gente mi odia, per il mio ridere. Perchè si riconosce. È orribile lo specchio quando si è diventati brutti, vecchi, stanchi. Quando finita è giovinezza" (T, 408). 
In racconti d'ambientazione fantastico-surrealistica Pirandello (ad es. "I due giganti") e Buzzati allacciano con la propria giovinezza e con la propria vecchiaia un dialogo che lo stile indiretto libero sembra tradurre in ripiegamenti di un monologo pateticamente umoristico, riuscendo simultaneamente a straniare e a riunire le due parti dimezzate dell' "io" autobiografico:

Stava ora dinanzi allo specchio, dritto e curvo, orgoglioso e sconfitto, padrone e servo, con quella brutta piega all' angolo dell'occhio,

E perchè così fermo? Cosa c'era? Qualche ricordo? [...] O il rimorso? $\mathrm{O}$ il pensiero di aver sbagliato tutto? $\mathrm{O}$ gli amici perduti? O i rimpianti?

Rimpianti di cosa? Della giovinezza che è finita per caso? Ma lui se ne ride della giovinezza, la giovinezza non gli ha dato che pene e malinconie. Lui se ne ride ah ah. Lui ha tutto ciò che l'uomo può onestamente desiderare. No, al tempo, rettifico. Non proprio tutto, anzi qualcosa soltanto. Anzi niente, adesso che ci penso.

A questo punto lo chiamai sporgendomi dal davanzale. Ciao, dissi, perchè era un vecchio amico. Lui neppure si voltò, con la destra fece un cenno quasi per dire andate andate [...] Cercava di tenersi su, con le mani sui fianchi, imbecille. E sorrideva anche. Ero io. (IC, 442-443, e cfr NA, II, 1210-1214)

Nel dramma buzzatiano Una ragazza arrivò c'è un'ampia scena favolosa, articolata con ritmi e cadenze della filastrocca, in cui Leonella, una sorta di Cenerentola concepita alla rovescia, dialoga con lo "Specchio", una sorta di mago con forti virtù miracolose, chiedendogli di non rivelarle più le bruttezze somatiche ("O specchio finiscila / il mattino di dirmi / che naso impossibile / alla sera di dirmi che guance flaccide / [...] che bocca insipida /che gambe a virgola") e di metamorfosarla in una bella fanciulla ( $\mathrm{T}, 226-227)$. Ma lo 
"Specchio" si rivela il suo antagonista perchè non è sua prassi nè di dire menzogne nè di alimentare sogni $(T, 227)$. Presa da un impeto di violenza, Leonella lo rompe in dodici "Specchietti". La giovane fanciulla subito li supplica di secondare le sue illusioni: "Vi prego mentite / barate fingete / la bellezza che non c'è [...] / Anche se sono un mostro / mi direte bugie / fra il lusco e il brusco / mi darete illusioni / sogni e fantasticherie / d'essere regina / con abito di lamé / ermellini e visioni / corona di stelle" (T, 228-229). E gli "Specchietti" profondamente impietositi, la secondano in tutto "così come vuo[le]. / Ti diremo le cose che non hai avute / le bugie dolci inganni / che fan battere il cuore" ( $T$, 228). Leonella si manifesta imparentata al personaggio pirandelliano femminile e non (ad es. Come tu mi voi, Enrico $I V$ ) che crede nelle proprie illusione, che le grida in faccia a tutti ("ci credo ci credo ci credo"), che le impone e le fa credere agli altri, e con una parlata dinamica che tesse una ragnatela contrasti $(T$, 229-231).

Nella weltanschauung buzzatiana i contrasti tra finzione e realtà, tra arte e vita, tra autore e personaggio si ripresentano con costanza, svelando un colorito pirandellismo, rifacimenti, echi, e affinità coi meta-racconti e con i meta-drammi di Pirandello. Certamente Buzzati non avrebbe scritto il dramma L'uomo che andrà in America in cui pittori, letterati, e attori disquisiscono della propria arte mentre l'opera si sviluppa, se non avesse avuto alle spalle e metabolizzata l'esperienza pirandelliana del teatro nel teatro, al punto che vi si suggerisce, come si fa nella "prefazione" ai Sei personaggi, che l'artista è un piccolo dio strumento delle forze della natura $(T, 389)$ e vi si lanciano pungenti critiche al mondo dell'arte contemporanea, come ad esempio fa Pirandello in Sei personaggi, Questa sera si recita a soggetto, L'innesto.

Come nelle opere pirandelliane (ad es. "La tragedia di un personaggio", "Fuoco alla paglia", Sei personaggi), in quelle buzzatiane ci sono presonaggi che appaiono nell'azione come se 
improvvisamente fossero piovuti dal cielo, in modo immediato e spontaneo, mettendosi in polemica e in contrasto con il loro creatore.

Nell'"Ombra dell'ammiraglio" Buzzati si presenta in veste di corrispondete di guerra. Dal momento che mette i piedi a bordo di una nave in missione, si sente molto rispettato. Dato che l'ammiraglio è assente, gli viene assegnato la camera di lui, descritta dettagliatamente in un periodo alquanto lungo. Rimane sbalordito appena la vede, con ogni comodità, bella, elegante, lussuosa. E ci si sente felice, un "papa", poichè può farci tutti i comodi come se fosse a "casa [sua], nell' albergo dell'Eccellenza" (IB, 35). Ma presto l'incanto delle cose viene infranto dal contrasto tra la figura borghese del giornalista e i marinai che svolgono un lavoro bestiale (IB, 37). Questo contrasto sottende anche la favola onirica che chiude la narrazione. Dove la figura dell'ammiraglio fa un'apparizione arieggiante a quella di Madame Pace dei Sei personaggi, si presenta pirandellianamente nella visione sognante di Buzzati, e gli si sdraia vicino rimproverandolo di dormire placidamente nel suo letto, ossia di condurre una bella vita: " "Voi, che cosa fate qui? [...] Voi dormite, eh? Nel mio letto dormite, vero? Il bel signorino! Ah, e che cosa aspetta? Fuori di qui!' Io balbettavo [...], e in cuor mio riconoscendo che egli aveva abbastanza ragione" (IB, 38). Insomma, come a un diverso livello fa il personaggio pirandelliano di "Colloqui coi personaggi" al suo creatore, questo personaggio rivolge una dura critica al Buzzati giornalista-scrittore incapace di portarsi fuori da certi abitudini di borghese; (il personaggio fantasma che pirandellianamente viene a dettare principi morali e critiche sociali, e a richiamare il suo demiurgo si ripresenta in altri racconti di Buzzati, cfr. ad es. "Ridestati per il Giro i fantasmi della vecchia Cassino").

In "Melinda, strega per forza" il Buzzati giornalista-scrittore sposta l'indagine nel mondo arcano dell'Abruzzo. A fargli da guida è il giornalista locale, l'amico Franco Manocchia. Questi ama parlargli della vita di certi personaggi da lui conosciuti. Come la strega Melinda. Buzzati è commosso dalla ricostruzione storico-memoriale 
che ne fa l'amico. Ed ecco che tutto a un tratto al giornalista-scrittore, attraverso la "servetta fantasia", gli si presenta inaspettatamente Melinda, come fanno certi personaggi pirandelliani che nascono in momenti prodigiosi della creazione (ad es. Sei personaggi); balza sulla scena e gli si impone a guisa di personaggio "vivo", in carne ed ossa, per motivarlo a raccontare una storia con più punti di vista, da quello di Manocchia a quello di Buzzati, a quello della stessa Melinda, visceralmente confessionale ed incapsulato in lunghi brani in corsivo frammetariamente intercalati nella narrazione. Pirandellianamente prende la mano dello scrittore-giornalista, presentandosi come la settima figlia di una famiglia senza maschi. Come tanti personaggi pirandelliani anche della realtà primitiva della Sicilia, la storia che narra è una storia emblematica di un individuo maledetto, di un essere soggiogato dalle mani di un destino crudele, simboleggiato dalla famiglia che la forza contro la propria voglia ad essere strega, e che allegorizza una società sprofondata in tradizioni millenarie, scongiuratrice e superstiziosa, gretta e stolta, dura e tirannica, che ferocemente schiaccia l'individuo spingendolo nell'ambito dell'ostracismo (MI, 110, e cfr. ad es. "Il libretto rosso", "Ciaula scopre la luna", "La lega disciolta" di Pirandello). Si tratta quindi di una storia sottesa dal messaggio morale identico a quello delle favole pirandelliane, in quanto il vero diavolo non è la strega Melinda, è invece una società che si crede composta di gente retta e magnanima. La voce narrante di Melina si fa dominante, si impone a esporre le cose con vitalismo simile a quello del personaggio pirandelliano che agogna di vendicarsi contro gli altri e il destino nemico (MI, 108). Ma a volte i giuochi pirandelliani danno l'impressione che la voce narrante di Manocchia sembri quella di Buzzati; l'uno è la maschera dell'altro e viceversa, e a livelli diversi si identificano col personaggio di Melinda, descritto in un contesto storico-antropologico. Quando Manocchia-Buzzati si dilata a rievocare gli intenti delle fatture, dei sortilegi, dei feticci, dell'operare di Melinda, questa balza sulla scena come volesse protestare, interrompe e prende la parola per rinforzare 
quella sua immagine di strega benigna, magnanima, giusta, al di sopra del proprio ambiente (MI, 110-112). Il paradosso si fa drammatico quando la strega, come certi stregoni di Pirandello (ad es. "La patente"), si erge una sorta di giudice amante della verità e della giustizia, e l'uomo una bestia brutale (MI, 111).

Invece in "Storia interrotta", come nel "Critico d'arte" ed altri racconti meta-creativi di Buzzati, si ha a che fare con un testo che pirandellianamente non si lascia scrivere. Si tratta di una "storia" incompiuta, parzialmente realizzata dall'autore anni addietro, ma che ora riprende con l'intento di terminarla. Durante la prima versione egli si sente pirandellianamente dominato dalla "storia" (sinonimo di personaggio) che sta creando, che essa gli prende la mano e va "per suo conto, non continuando a obberdir[lo], bensì sviluppandosi a caso" (ND, 103). Allora, come adesso, il rapporto tra la storia(-personaggio) e il suo creatore si fa drammaticamente conflittuale. Ricorda che a un tratto gli si esaurisce, un'azione che fa richiamare certi meta-personaggi pirandelliani che rifiutano il loro autore (ad es. "Colloqui coi personaggi"). Ora non la sente più sua: "un altro dunque l'aveva inventata, un uomo diverso da me, estraneo; e scomparso per sempre" (ND, 103). E rileggendone alcune pagine informa il lettore di aver abbozzato la descrizione di un paesaggio primaverile e di alcuni personaggi, tra cui si impone la figura della giovane Marietta che dolcemente "canta" alla finestra e l'arrivo di un misterioso cavaliere. Con la comparsa del cavaliere finisce la storia stesa tempo fa.

Tuttavia il cavaliere si modella una figura di personaggio indomabile ed autonomo come Leandro Scotto, il dottor Fileno e altre creature pirandelliane, che apparisce e sparisce nella storia in progresso, denotando il mistero della creazioni, dell'ispirazione che va e viene e non si può controllare, e vari significati del rapporto tra l'arte e il tempo (ND, 101, cfr. ad es. "Colloquio coi personaggi" di Pirandello).

Come in Pirandello (ad es. "Colloqui coi personaggi", "Personaggi", "La tragedia di un personaggio"), lo scrittore Buzzati si 
mette a dialogare con le sue incompiute creature, in particolare con Marietta, che è un'immagine opposta a quella del cavaliere, di personaggio che non si ribella, remissivo e disposto a secondare l'esigenze dell'estro creativo dell'autore. Ma ciononostante Buzzati non riesce a completare "la storia interrotta": "morta è dunque la vecchia storia dentro di me, senza che me ne accorgessi; rimasta rotta a metà; e oggi è tardi per ricominciare" (ND, 108).

Buzzati fa sentire la ricalcatura conscia e inconscia del mondo pirandelliano quando sviluppa un ossesionante discorso di meta-scrittura, pervaso da sogni e ideali di scrivere grandi capolavori (ad es. "Meraviglioso mestire" di Buzzati; Malgiocondo di Pirandello), e infranti da crisi di mancanza d'ispirazione e di sterilità creativa, come illustra la realizzazione del personaggio artista in declino che ha rapporto difficili anche con il suo editore, in Quando si è qualcuno di Pirandello e in Drammatica fine di un noto musicista di Buzzati. O quando conduce agli estremi gli sperimentalismi relativi alla composizione del testo teatrale, da Sola in casa a Una ragazza arrivò in cui l'immagine di Leonella attrice (che sta provando una pièce da mettere in scena) sembra modellata sull'attrice pirandelliana del "Pipistrello" e di Trovarsi, anche per l'abilità di Leonella di stregare gli spettatori durante la recita, e i suoi conflitti con il "Direttore" si modellano su quelli che emergono tra il "capocomico" e gli attori nei meta-drammi pirandelliani (ad es. Sei personaggi, Questa sera si recita a soggetto), e i conflitti tra gli astanti ricalcano quelli che Pirandello rappresenta in Ciascuno a suo modo (T, 240-252). O quando si cimenta a esplorare i paradossi dell'esistenza e quelli di una forza bizzarra che può cambiare in qualsiasi attimo la vita dell'individuo, come mostra "Sic transit" che ritrae la storia di un ministro che inaspettatamente ridiventa l'uomo che era prima della ascesa sociale, l'uomo povero che faceva pulizie per sopravvivere, paradossi che in Pirandello e in Buzzati si costruiscono con immagini di animali, come avverte il racconto buzzatiano "I topi", in cui i gatti che vengono messi per mangiare i topi che hanno invaso un'abitazione si rivelano 
inefficienti perchè sono molto più piccoli dei topi che dovrebbero annullare, una situazione più o meno simile a quella dell'apertura del Fu Mattia Pascal. O quando enfatizza certe fissazioni, idee, visioni, come quelle che hanno a che fare con il relativismo della realtà ("La frana" CB, 324-325), con la vita che "è una cosa ben buffa" (SS, 491), con le assurdità delle azioni umane (ad es. "La casa dell'abate").

Tutti i parallelismi, le comunanze, gli avvicinamenti che si possono realizzare delle opere di questi due scrittori suggeriscono che essi hanno una visione fondamentalmente pessimistica dell'esistenza (cfr. ad es. "Weekend" di Buzzati e "Il professor Terremoto" di Pirandello), che per loro la realtà è profondamente misteriosa, e che il pirandellismo di Buzzati, come quello di grandi scrittori, da Bontempelli a Borges, da Cortázar a Landolfi a Calvino ${ }^{10}$, presenta profonda originalità.

(Baruch College, City University of New York)

9 Si veda D. Buzzati, In quel preciso momento, Milano, Mondadori, 1963: 94-96.

10 Per il pirandellismo di questi e di altri scrittori contemporanei si rimanda ai miei interventi in Linea pirandelliana nella narrativa contemporanea, cit., e in Pirandello nell'America Latina, cit. 